\title{
Vortices and Localization in Euler Flows *
}

\author{
Carlo Marchioro and Mario Pulvirenti \\ Dipartimento di Matematica, Università "La Sapienza”, Piazzale A. Moro 2, I-00185 Roma, Italy
}

Received June 15, 1992; in revised form November 11, 1992

\begin{abstract}
We study the time evolution of a non-viscous incompressible two-dimensional fluid when the initial vorticity is concentrated in $N$ small disjoint regions of diameter $\varepsilon$. We prove that the time evolved vorticity is also concentrated in $N$ regions of diameter $d$, vanishing as $\varepsilon \rightarrow 0$. As a consequence we give a rigorous proof of the validity of the point vortex system. The same problem is examined in the context of the vortex-wave system.
\end{abstract}

\section{Introduction}

This paper is devoted to the study of the behavior of the time evolution of a nonviscous incompressible two-dimensional fluid, when the initial data becomes singular. Namely we study the case in which the initial vorticity is sharply concentrated in $N$ small disjoint regions of diameter $\varepsilon$. We prove that the time evolved vorticity is also concentrated in $N$ small regions. More precisely we prove that, with the total vorticity of each region fixed and an arbitrary time $t>0$, the support of the vorticity is contained in $N$ disjoint disks of radius $d, d$ vanishing with $\varepsilon$. We call this property "localization."

The difficulty in proving this localization property relies in the divergent kernel describing the interaction among the vorticity elements. Actually, when $\varepsilon$ is very small, the velocity field in each blob becomes very large and it is difficult to exclude that the radial component of the velocity pushes away thin filaments of vorticity. We prove that this does not happen.

At the same time, as a main consequence of the present result, we prove a general rigorous connection between the Euler Equation and the point vortex theory (for the first definition of the point vortex system see [1], for a review on the topic see [2,3]).

\footnotetext{
* Research partially supported by MURST, (Ministero dell'Università e della Ricerca Scientifica e Tecnologica), CNR (Consiglio Nazionale delle Ricerche - Gruppo Nazionale per la Fisica Matematica) and CNR contract n.92.00544.01
} 
Namely we prove that the vortex system describes the asymptotic behavior $\varepsilon \rightarrow 0$ of the Euler flow.

Partial results have been previously obtained for short time and vortex intensity of every sign in [4], for any time and vortices of the same sign in [5]. (Furthermore there are two particular results globally in time: one vortex [6] and two vortices of different sign [7] both cases in bounded regions.)

Finally, we note that the localization property was already proved for the simple system composed by one vortex only in the absence of any external field [5]. The main statement of the present paper is a non-trivial improvement of such a result.

In Sect. 2 we establish the main result, which will be proved in Sect. 3. Then in Sect. 4 we discuss possible generalizations when the initial data do not have compact support. Finally in Sect. 5 we apply the result of Sect. 2 to the vortex-wave system.

\section{Main Results}

Consider the Euler Equation in $\mathbb{R}^{2}$ in terms of vorticity:

$$
\begin{gathered}
\partial_{t} \omega(x, t)+(u \cdot \nabla) \omega(x, t)=0, \\
\nabla \cdot u(x, t)=0, \\
\omega \equiv \operatorname{curl} u \equiv \partial_{1} u_{2}-\partial_{2} u_{1}, \quad \omega(x, t)=\omega_{0}, \quad x=\left(x_{1}, x_{2}\right) .
\end{gathered}
$$

Here $u=\left(u_{1}, u_{2}\right)$ denotes the velocity field.

If $u$ decays at infinity, we can reconstruct the velocity field by means of $\omega$ as

$$
\begin{gathered}
u(x, t)=\int K(x-y) \omega(y, t) d y, \\
K=\nabla^{\perp} G, \\
\nabla^{\perp} \equiv\left(\partial_{2},-\partial_{1}\right), \\
G(x)=-\frac{1}{2 \pi} \ln |x| .
\end{gathered}
$$

As is well known, Eq. (2.1) means that the vorticity is constant along the particle paths which are the characteristics of the Euler equations. Therefore

$$
\omega(x, t)=\omega\left(x_{0}(x,-t), 0\right),
$$

where the trajectory $x\left(x_{0}, t\right)$ of the fluid particle, initially in $x_{0}$, satisfies:

$$
\begin{gathered}
\frac{d}{d t} x\left(x_{0}, t\right)=u\left(x\left(x_{0}, t\right), t\right), \quad x\left(x_{0}, 0\right)=x_{0}, \\
u(x, t)=\int K(x-y) \omega(y, t) d y .
\end{gathered}
$$

As is well known Eq. (2.8), (2.9), (2.10) imply the weak form of the Euler Equation:

$$
\frac{d}{d t} \omega[f]=\omega[u \cdot \nabla f]+\omega\left[\partial_{t} f\right]
$$

where $f(x, t)$ is a bounded smooth function and

$$
\omega[f] \equiv \int d x \omega(x, t) f(x, t) .
$$


It is well known that there exists a unique solution $\omega(x, t) \in L_{1} \cap L_{\infty}$ to the initial value problem associated to (2.11) provided that $\omega(x, 0) \in L_{1} \cap L_{\infty}$. Moreover the divergence-free condition (2.2) implies that the time evolution (2.9) preserves the Lebesgue measure on $\mathbb{R}^{2}$.

We consider an initial datum of the form:

$$
\omega_{\varepsilon}(x, 0)=\sum_{i=1}^{N} \omega_{\varepsilon ; 2}(x, 0),
$$

where $\omega_{\varepsilon ; 2}(x, 0)$ is a function with a definite sign supported in a region $\Lambda_{\varepsilon ; i}$ such that

$$
\Lambda_{\varepsilon ; \imath} \equiv \operatorname{supp} \omega_{\varepsilon ; \imath} \subset \Sigma\left(z_{i} \mid \varepsilon\right) ; \quad \Sigma\left(z_{i} \mid \varepsilon\right) \cap \Sigma\left(z_{\jmath} \mid \varepsilon\right)=\emptyset \quad \text { if } \quad i \neq j
$$

for $\varepsilon$ small enough, where $\Sigma(z \mid r)$ is the circle of center $z$ and radius $r$.

We denote by

$$
\int d x \omega_{\varepsilon ; i}(x, 0)=a_{\imath} \in \mathbb{R}
$$

the vortex intensity (independent of $\varepsilon$ ) and we assume

$$
\left|\omega_{\varepsilon ; i}(x, 0)\right| \leq \operatorname{const} \varepsilon^{-\eta}, \quad \eta<\frac{8}{3} .
$$

A particular case considered in previous papers satisfying (2.15) and (2.16) is given by:

$$
\omega_{\varepsilon ; i}(x, 0)=a_{i} \varepsilon^{-2} \chi\left(\Lambda_{\varepsilon ; i}\right),
$$

where $\chi(\Lambda)$ denotes the characteristic function of the set $\Lambda$ and

$$
\text { meas } \Lambda_{\varepsilon ; 2}=\varepsilon^{2} \text {. }
$$

We prove the following result:

Theorem 2.1. Denote by $\omega_{\varepsilon}(x, t)$ the time evolution of $\omega_{\varepsilon}(x, 0)$ according to the Euler Equation. Then, for a fixed arbitrary $T>0$,

i) for all $d>0$ there exists $\varepsilon_{0}(d, T)$ such that, if $\varepsilon<\varepsilon_{0}(d, T)$, then

$$
\operatorname{supp} \omega_{\varepsilon ; i}(x, t) \subset \Sigma\left(z_{i}(t) \mid d\right) \text { for any } t \in[0, T] \text {, }
$$

where $z_{i}(t)$ is the solution of the ordinary differential system (called point vortex system)

$$
\begin{gathered}
\frac{d}{d t} z_{\imath}(t)=-\nabla_{i}^{\perp} \frac{1}{2 \pi} \sum_{j=1 ; j \neq i}^{N} \ln \left|z_{i}(t)-z_{\jmath}(t)\right|, \\
z_{\imath}(0)=z_{i},
\end{gathered}
$$

provided that such a solution exists up to the time T. Moreover $d \rightarrow 0$ as $\varepsilon \rightarrow 0$.

ii) For any continuous bounded function $f(x)$,

$$
\lim _{\varepsilon \rightarrow 0} \int d x \omega_{\varepsilon}(x, t) f(x)=\sum_{\imath=1}^{N} a_{\imath} f\left(z_{i}(t)\right) .
$$

The proof will be given in the next section. Here we briefly comment on the statements of the theorem. i) states that the blobs of vorticity remain localized until time $T$ for any $d$ and $T$, provided that we choose $\varepsilon$ small enough. ii) states that

$$
\omega_{\varepsilon}(x, t) \underset{\varepsilon \rightarrow 0}{\longrightarrow} \sum_{i=1}^{N} a_{\imath} \delta\left(z_{\imath}(t)\right)
$$


weakly in the sense of measures, where $\delta(\cdot)$ denotes the Dirac measure. This last statement gives a rigorous justification of the point vortex model.

We observe that the singular nature of the right-hand side of Eq. (2.20), diverging when two vortices are close to each other, does not guarantee the existence of the solutions of Eq. (2.20) for every time. In many cases (for instance for $a_{\imath}>0$ ) collapses are forbidden by the first integrals of the motion, but there are cases in which singularities do happen. However it can be proved that the collapses are exceptional [2] in the sense that the initial configurations developing singularities have Lebesgue measure zero. In general we can either restrict ourselves to this full measure set or say that theorem 2.1 holds up to the time $T$ for which the solutions of Eq. (2.20) exist.

\section{Proof of Theorem 2.1}

First we consider a single blob of unitary vorticity moving in an external, divergencefree, uniformly bounded, time dependent, vector field $F(x, t)$, satisfying the Lipschitz condition

$$
|F(x, t)-F(y, t)| \leq L|x-y|, \quad L>0 .
$$

Equation (2.9) becomes

$$
\frac{d}{d t} x(t)=u(x, t)+F(x, t),
$$

while Eqs. (2.8), (2.10) remain unchanged. The Euler equation in weak form reads

$$
\frac{d}{d t} \omega[f]=\omega[(u+F) \cdot \nabla f]+\omega\left[\partial_{t} f\right] .
$$

Then we prove Proposition i) of Theorem 2.1 for this particular evolution. Define the center of vorticity as

$$
B_{\varepsilon}(t)=\int x \omega_{\varepsilon}(x, t) d x .
$$

Theorem 3.1. Suppose that

$$
\operatorname{supp} \omega_{\varepsilon}(x, 0) \subset \Sigma\left(x^{*} \mid \varepsilon\right) \quad \text { and }\left|\omega_{\varepsilon}(x, 0)\right| \leq \text { const } \varepsilon^{-\eta,} \quad \eta<\frac{8}{3},
$$

and fix an arbitrary $T>0$. Then for any $d>0$, there exists $\varepsilon_{0}(d, T)>0$ such that, if $\varepsilon<\varepsilon_{0}$, we have:

$$
\operatorname{supp} \omega_{\varepsilon}(x, t) \subset \Sigma(B(t) \mid d) \text { for any } t \in[0, T],
$$

where $B(t)$ is the solution of the ordinary differential equation

$$
\frac{d}{d t} B(t)=F(B(t), t), \quad B(0)=x^{*} .
$$

Moreover

$$
\lim _{\varepsilon \rightarrow 0} B_{\varepsilon}(t)=B(t) \quad \text { uniformly in } t \in[0, T] .
$$

Remark. The above theorem does not assert that the motion of the fluid particles supporting the vorticity $\omega_{\varepsilon}$ converges, in the limit $\varepsilon \rightarrow 0$, to $B(t)$. In general, this is false. The motion of such fluid particles, due to the singularity of the kernel $K(x-y)$, 
is very irregular and is not converging at all. However the motion of the center of vorticity converges to the motion of a single point vortex in the velocity field $F$.

The above theorem is a basic preliminary step in proving the validity of the vortex model: we are looking to the behaviour of a single vortex, assuming that the field generated by all the others is given and smooth.

Proof. The difficulty of the proof arises from the singularity of the kernel $K$ which forces a fluid particle rotate with a very large velocity around the center of vorticity. To overcome this difficulty we study the motion of the center of vorticity which will turn out to be much more regular than the motion of a given fluid particle. Moreover, the moment of inertia is almost conserved during the motion, so that we can also control the spreading of the vorticity distribution around the center of vorticity. However, as we shall see, the control given by the moment of inertia is not enough for our purposes.

We introduce the moment of inertia $I_{\varepsilon}$ with respect to $B_{\varepsilon}$ :

$$
I_{\varepsilon}(t)=\int \omega_{\varepsilon}(x, t)\left(x-B_{\varepsilon}(t)\right)^{2} d x
$$

Then we study its growth in time. If $F$ would vanish, $B_{\varepsilon}$ and $I_{\varepsilon}$ would be constant along the motion. For $F \neq 0$ we have

$$
\begin{gathered}
\frac{d}{d t} B_{\varepsilon}(t)=\int F(x, t) \omega_{\varepsilon}(x, t) d x \\
\frac{d}{d t} I_{\varepsilon}(t)=2 \int\left(x-B_{\varepsilon}(t)\right) \cdot F(x, t) \omega_{\varepsilon}(x, t) d x,
\end{gathered}
$$

where we have taken into account the antisymmetry of $K$. Making use of the fact that

$$
\int\left(x-B_{\varepsilon}(t)\right) \cdot F\left(B_{\varepsilon}(t), t\right) \omega_{\varepsilon}(x, t) d x=0
$$

and the Lipschitz condition on $F$, we have

$$
\left|\frac{d}{d t} I_{\varepsilon}(t)\right| \leq 2 L \int \omega_{\varepsilon}(x, t)\left(x-B_{\varepsilon}(t)\right)^{2} d x=2 L I_{\varepsilon}(t)
$$

from which

$$
I_{\varepsilon}(t) \leq I_{\varepsilon}(0) \exp (2 L t)
$$

Therefore $\lim _{\varepsilon \rightarrow 0} I_{\varepsilon}(t)=0$, uniformly in $t \in[0, T]$, since

$$
\begin{aligned}
I_{\varepsilon}(0) & =\int \omega_{\varepsilon}(x, 0)\left(x-x^{*}\right)^{2} d x \\
& =\int_{\Sigma\left(x^{*} \mid \varepsilon\right)} \omega_{\varepsilon}(x, 0)\left(x-x^{*}\right)^{2} d x<\varepsilon^{2} \rightarrow 0 \quad \text { as } \varepsilon \rightarrow 0 .
\end{aligned}
$$


Then it is easy to prove Eq. (3.8). In fact

$$
\begin{aligned}
\left|B(t)-B_{\varepsilon}(t)\right| \leq & \left|x^{*}-B_{\varepsilon}(0)\right|+\int_{0}^{t} d s\left|F(B(s), s)-\int d x F(x, s) \omega_{\varepsilon}(x, s)\right| \\
\leq & \left|x^{*}-B_{\varepsilon}(0)\right|+\int_{0}^{t} d s\left|F(B(s), s)-F\left(B_{\varepsilon}(s), s\right)\right| \\
& +\int_{0}^{t} d s\left|F\left(B_{\varepsilon}(s), s\right)-\int d x F(x, s) \omega_{\varepsilon}(x, s)\right| \\
\leq & \left|x^{*}-B_{\varepsilon}(0)\right|+L \int_{0}^{t} d s\left|B(s)-B_{\varepsilon}(s)\right| \\
& \left.+L \int_{0}^{t} d s \int d x \mid B_{\varepsilon}(s), s\right)-x \mid \omega_{\varepsilon}(x, t) \\
\leq & \left|x^{*}-B_{\varepsilon}(0)\right|+L \int_{0}^{t} d s\left|B(s)-B_{\varepsilon}(s)\right| \\
& +L T \sup _{0 \leq t \leq T} \sqrt{I_{\varepsilon}(t)} .
\end{aligned}
$$

By the Gronwall Lemma, because the first and the third term in the right-hand side of (3.16) are vanishing in the limit $\varepsilon \rightarrow 0$, we finally achieve the proof of statement (3.8).

We now proceed in proving the last step, namely statement (3.6). We study the vorticity amount crossing the boundary of a small disk around $B_{\varepsilon}(t)$. We prove that it is small and so the radial part of the velocity field is also small and the particle paths cannot go far apart from $B_{\varepsilon}$.

To control the vorticity flux we introduce, for $R>0$, the following function $W_{R} \in C^{\infty}\left(\mathbb{R}^{2}\right), r \rightarrow W_{R}(r)$ depending only on $|r|$, defined as:

$$
W_{R}(r)= \begin{cases}1 & \text { if }|r| \leq R \\ 0 & \text { if }|r|>2 R\end{cases}
$$

such that, for some $C_{1}>0$ :

$$
\begin{gathered}
\left|\nabla W_{R}(r)\right| \leq \frac{C_{1}}{R} \\
\left|\nabla W_{R}(r)-\nabla W_{R}\left(r^{\prime}\right)\right| \leq \frac{C_{1}}{R^{2}}\left|r-r^{\prime}\right| .
\end{gathered}
$$

Define the quantity:

$$
\mu_{R}(t)=1-\int d x W_{R}\left(x-B_{\varepsilon}(t)\right) \omega_{\varepsilon}(x, t) .
$$


Notice that, if $\omega_{\varepsilon}(x, t) \subset \Sigma\left(B_{\varepsilon}(t), R\right)$ then $\mu_{R}(t)=0$. Thus we choose $\mu_{R}$ as a measure of the localization of $\omega_{\varepsilon}(x, t)$ around $B_{\varepsilon}$. Then we evaluate the time derivative by using (3.3):

$$
\begin{aligned}
\frac{d \mu_{R}(t)}{d t}= & -\int d x \nabla W_{R}\left(x-B_{\varepsilon}(t) \cdot\left\{u(x, t)+F(x, t)-\frac{d}{d t} B_{\varepsilon}(t)\right\} \omega_{\varepsilon}(x, t)\right. \\
= & \int d x \omega_{\varepsilon}(x, t) \nabla W_{R}\left(x-B_{\varepsilon}(t)\right) \cdot \int d y K(x-y) \omega_{\varepsilon}(y, t) \\
& -\int d x \omega_{\varepsilon}(x, t) \nabla W_{R}\left(x-B_{\varepsilon}(t)\right) \\
& \times \int d y \omega_{\varepsilon}(y, t)[F(x, t)-F(y, t)] .
\end{aligned}
$$

We now estimate the first term in the right-hand side of Eq. (3.21). By the antisymmetry of $K$, it can be written as:

$$
\begin{aligned}
& -\frac{1}{2} \int d x \int d y \omega_{\varepsilon}(x, t) \omega_{\varepsilon}(y, t) \\
& \quad \times\left\{\nabla W_{R}\left(x-B_{\varepsilon}(t)\right)-\nabla W_{R}\left(y-B_{\varepsilon}(t)\right)\right\} \cdot K(x-y) .
\end{aligned}
$$

To estimate this term we split the integration domain in the following sets:

$$
\begin{aligned}
& T_{1}=\left\{(x, y) \mid x \in \Sigma^{c}\left(B_{\varepsilon}(t) \mid R\right) y \in \Sigma\left(B_{\varepsilon}(t) \mid \gamma\right\},\right. \\
& T_{2}=\left\{(x, y) \mid x \in \Sigma^{c}\left(B_{\varepsilon}(t) \mid R\right) y \notin \Sigma\left(B_{\varepsilon}(t) \mid \gamma\right\},\right. \\
& T_{3}=\left\{(x, y) \mid y \in \Sigma^{c}\left(B_{\varepsilon}(t) \mid R\right) x \in \Sigma\left(B_{\varepsilon}(t) \mid \gamma\right\},\right. \\
& T_{4}=\left\{(x, y) \mid y \in \Sigma^{c}\left(B_{\varepsilon}(t) \mid R\right) x \notin \Sigma\left(B_{\varepsilon}(t) \mid \gamma\right\},\right.
\end{aligned}
$$

where $\gamma=R^{\alpha}$ ( $\alpha>1$ will be fixed later) and, from now on, $R<\frac{1}{2}$ so that $\gamma<R$.

Notice that the integrand in (2.22) vanishes in the complement of $T_{1} \cup T_{2} \cup T_{3} \cup T_{4}$.

Thanks to the identities $\nabla W_{R}\left(x-B_{\varepsilon}(t)\right) \cdot K\left(x-B_{\varepsilon}(t)\right)=0$ and $\nabla W_{R}\left(y-B_{\varepsilon}(t)\right)=$ 0 if $y \in \Sigma\left(B_{\varepsilon}(t) \mid \gamma\right)$, the contribution of the integral (3.22) due to $T_{1}$ is bounded by

$$
\frac{1}{2} \mid \int d x \int_{\Sigma\left(B_{\varepsilon}(t) \mid \gamma\right)} d y \omega_{\varepsilon}(x, t) \omega_{\varepsilon}(y, t) \nabla W_{R}\left(x-B_{\varepsilon}(t)\right) \cdot\left\{K(x-y)-K\left(x-B_{\varepsilon}(t)\right\} \mid\right.
$$

$\leq$ by (3.18) and the fact that $\nabla W_{R}\left(x-B_{\varepsilon}(t)\right)=0$ if $\left.\left|x-B_{\varepsilon}(t)\right|<R\right]$

$$
\begin{aligned}
& \leq \frac{C_{1}}{2}\left|\int d x \int_{\left(B_{\varepsilon}(t) \mid \gamma\right)} d y \omega_{\varepsilon}(x, t) \omega_{\varepsilon}(y, t) \frac{\left|y-B_{\varepsilon}(t)\right|}{R(R-\gamma)^{2}} \chi\left(\left|B_{\varepsilon}(t)-y\right|>R\right)\right| \\
& \leq \frac{C_{1}}{2} m_{t}(R) \frac{\gamma}{R(R-\gamma)^{2}} \leq C_{2} m_{t}(R) R^{\alpha-3} .
\end{aligned}
$$

Here we set:

$$
m_{t}(R)=\int_{\Sigma^{c}\left(B_{\varepsilon}(t) \mid R\right)} \omega_{\varepsilon}(x, t) d x,
$$

that is the amount of vorticity outside $\Sigma\left(B_{\varepsilon}(t), R\right)$.

To estimate the contribution due to $T_{2}$ we use, thanks to the obvious inequality $|K(x)| \leq C|x|^{-1},(3.19)$ and the bound:

$$
\left|\left\{\nabla W_{R}\left(x-B_{\varepsilon}(t)\right)-\nabla W_{R}\left(y-B_{\varepsilon}(t)\right)\right\} \cdot K(x-y)\right| \leq C_{3} R^{-2}
$$


from which we estimate such a contribution by:

$$
C_{4} \frac{m_{t}(\gamma) m_{t}(R)}{R^{2}}
$$

The contributions due to $T_{3}$ and $T_{4}$ can be handled exactly in the same way.

To achieve the estimate of the time derivative of $\mu_{R}$, we evaluate the second integral in the right-hand side of Eq. (3.21). It is:

$$
-\int d x \omega_{\varepsilon}(x, t) \nabla W_{R}\left(x-B_{\varepsilon}(t)\right) \cdot \int d y \omega_{\varepsilon}(y, t)\{F(x, t)-F(y, t)\} .
$$

We split the domain of integration in $y$ into the two regions $\left|y-B_{\varepsilon}(t)\right|>R$ and its complement. The first contribution is bounded by:

$$
2\|F\|_{\infty} \frac{m_{t}(R)^{2}}{R}
$$

while the second one is certainly bounded by:

$$
C_{5} m_{t}(R)
$$

since from Eq. (3.18),

$$
\left|\nabla W_{R}\left(x-B_{\varepsilon}(t)\right)\right||x-y| \leq \text { Const } .
$$

Before collecting all the above estimates we evaluate $m_{t}(R)$ in terms of $I_{\varepsilon}(t)$ :

$$
m_{t}(R) \leq \frac{1}{R^{2}} \int_{\Sigma\left(B_{\varepsilon}(t) \mid R\right)} d x \omega_{\varepsilon}(x, t) x^{2} \leq \frac{I_{\varepsilon}(t)}{R^{2}} \leq C_{6} \frac{\varepsilon^{2}}{R^{2}}
$$

[here we used (3.14)]. In conclusion

$$
\left|\frac{d \mu_{R}}{d t}\right| \leq C_{5} m_{t}(R)+A(R, \varepsilon),
$$

where

$$
A(R, \varepsilon)=C_{7} \varepsilon^{2}\left(R^{\alpha-5}+\varepsilon^{2} R^{-2 \alpha-4}+\varepsilon^{2} R^{-5}\right) .
$$

On the other hand, we can bound $m_{t}(R)$ in terms of $\mu_{R / 2}$ :

$$
m_{t}(R)=1-\int_{\Sigma\left(B_{\varepsilon}(t) \mid R\right)} d x \omega_{\varepsilon}(x, t) \leq 1-\int d x \omega_{\varepsilon}(x, t) W_{R / 2}\left(x-B_{\varepsilon}(t)\right)
$$

so that from (3.32) we arrive at the integral inequality:

$$
\mu_{R}(t) \leq T A(R, \varepsilon)+C_{5} \int_{0}^{t} d \tau \mu_{R / 2}(\tau)+\mu_{R}(0) .
$$

The term $\mu_{R}(0)$ vanishes if $R>\varepsilon$.

We iterate inequality (3.35) $k$-times, with $k$ satisfying the conditions

$$
2^{-k} R>\varepsilon \text {. }
$$


We choose $R=\varepsilon^{\delta}, \delta<\frac{1}{2}$, and $k=$ integer part of $D|\ln \varepsilon|, D<\frac{1}{3}$ so that Eq. (3.36) is satisfied for $\varepsilon<1$. Therefore:

$$
\mu_{R}(t) \leq \frac{C_{5}^{k} t^{k}}{k !}+\sum_{s=1}^{k-1} T^{s+1} C_{5}^{s} A\left(R 2^{-s}, \varepsilon\right) .
$$

We see now that the first term in the right-hand side of inequality (3.37) can be bounded by $\varepsilon^{-\xi}$ with $\xi$ arbitrary large provide that $\varepsilon$ is small enough. This follows by observing that $k !>k^{k} e^{-k}$. Moreover the sum in (3.37) can be bounded by [see Eq. (3.33)]:

$$
\varepsilon^{-D \log C} \varepsilon^{2}\left(\varepsilon^{\delta \alpha-5 \delta}+\varepsilon^{2-2 \delta \alpha-4 \delta-(2 \alpha+4) D \log 2}+\varepsilon^{2-5 \delta-5 D \log 2}\right) .
$$

From this expression we realize that, choosing $D$ and $\delta$ small and $\alpha$ large, such that $\alpha \delta=\frac{2}{3}$, we have

$$
\mu_{R}(t) \leq \operatorname{const} \varepsilon^{\beta}
$$

with $\beta<\frac{8}{3}$ but arbitrary close to $\frac{8}{3}$. The definitive choice of $D, \delta, \alpha$, and consequently $\beta$ will be done later on.

So we have proved that the amount of vorticity escaping from the disk $\Sigma\left(B_{\varepsilon}(t), \varepsilon^{\delta}\right)$ is vanishing at least as $\varepsilon^{\beta}$. This information allows us to conclude the proof. Consider the disk $\Sigma_{2}=\Sigma\left(B_{\varepsilon}(t) \mid \varepsilon^{\delta / 3}\right)$. A particle localized in $x$, outside the boundary of such a disk, is moving under the action of three fields: the one generated by the vorticity inside the disk $\Sigma\left(B_{\varepsilon}(t) \mid \varepsilon^{\delta}\right)=\Sigma_{1}$, say $u_{1}$, the other one, $u_{2}$, generated by the vorticity outside the disk $\Sigma_{1}$ and $u_{3}$ due to the external field. Let $n$ be the versor in the direction $B_{\varepsilon}(t)-x$. Then:

$$
\begin{aligned}
\left|u_{1}(x) \cdot n\right| & =\left|n \cdot \int_{\Sigma_{1}} d y \omega_{\varepsilon}(y, t) K(x-y)\right| \\
& =\left|n \cdot \int_{\Sigma_{1}} d y \omega_{\varepsilon}(y, t)\left\{K(x-y)-K\left(x-B_{\varepsilon}(t)\right)\right\}\right| \\
& \leq C_{11} \frac{\varepsilon^{\delta}}{\left(\varepsilon^{\delta / 3}-\varepsilon^{\delta}\right)^{2}} \rightarrow 0 \quad \text { as } \quad \varepsilon \rightarrow 0 .
\end{aligned}
$$

Moreover

$$
\begin{aligned}
\left|u_{2}(x)\right| & =\left|\int_{\Sigma_{1}^{c}} d y \omega_{\varepsilon}(y, t) K(x-y)\right| \leq \int_{\Sigma_{1}^{c}} d y \frac{\omega_{\varepsilon}(y, t)}{|x-y|} \\
& \leq C \varepsilon^{-\eta} \int_{\Sigma(0 \mid r)} d y \frac{1}{|y|},
\end{aligned}
$$

where $r$ is defined by

$$
C \varepsilon^{-\eta} \pi r^{2}=m_{t}\left(\varepsilon^{\delta}\right)
$$

The last inequality (3.41) is a consequence of the fact that $\left|u_{2}(x)\right|$, among all the vorticity distributions $\omega_{\varepsilon}(y, t) \chi\left(\Sigma_{1}^{c}\right)$, bounded by $C \varepsilon^{-\eta}$ and whose total mass is given by $m_{t}\left(\varepsilon^{\delta}\right)$, is maximized by a constant vorticity distribution, valued $C \varepsilon^{-\eta}$, on a circle of radius $r$ [given by Eq. (3.42)]. 
By Eq. (3.42),

$$
r \leq C \varepsilon^{\beta / 2+\eta / 2}
$$

from which:

$$
\begin{gathered}
\left|u_{2}(x)\right| \leq \operatorname{const} \varepsilon^{-\eta} \int_{\Sigma(0 \mid r)} d y \frac{1}{|y|}=\text { const } \varepsilon^{\beta / 2-\eta / 2} \rightarrow 0, \\
\text { as } \varepsilon \rightarrow 0 \text { if } \beta>\eta .
\end{gathered}
$$

Equation (3.44) fixes the choice of $\beta: \beta \in\left(\eta, \frac{8}{3}\right)$.

Finally the Lipschitz condition on the external field assures that $u_{3} \rightarrow 0$ as $\varepsilon \rightarrow 0$.

Therefore the fields which could be responsible for bringing the particle paths far from $B_{\varepsilon}(t)$ are arbitrary small and it follows that in a finite time $T, \operatorname{supp} \omega_{\varepsilon}(x, t)$ must be contained in a fixed circle $\Sigma\left(B_{\varepsilon}(t) \mid d\right)$, for an arbitrary $d$, provided that $\varepsilon$ is sufficiently small. Then the proof of the theorem follows immediately by observing that $B_{\varepsilon}(t) \rightarrow B(t)$ as $\varepsilon \rightarrow 0$.

Remark. Notice that estimate (3.14) on the momentum of inertia is not enough to prove that $u_{2}$ is vanishing. Actually it gives only that the amount of vorticity outside a disk of a fixed radius $d$ around $B_{\varepsilon}(t)$ is of order $\varepsilon^{2}$. It generates a bounded field not vanishing a priori. Thus we need a more sophisticated analysis to prove the complete localization.

We give now the proof of Theorem 2.1 which, after Theorem 3.1 is almost obvious. Single out a blob, and consider the action of the others on it as an external perturbation field. If all the other blobs are rather far apart, they generate a Lipschitz vector field. On the other hand we proved that the blobs remain localized. Thus it is not difficult to achieve the program.

The proof follows readily by the following considerations. Let $b$ be the minimal distance at which any couple of point vortices can arrive in the time $T$, according to the vortex dynamics, for the initial datum $\left\{x_{i}\right\}_{\imath=1, N}$. Consider, for the initial condition $\omega_{\varepsilon}$ (with $\varepsilon$ to be chosen later) the following regularized dynamics for the Euler equation: two disjoint blobs interact via a kernel $K_{\eta}, \eta=\frac{b}{10} \cdot K_{\eta}$ is defined as a $C^{\infty}$ function which coincides with $K(x)$ when $|x| \geq \eta$. Each blob interacts with itself via the singular $K$. By Theorem 3.1 it is not difficult to prove Theorem 2.1 with the Euler dynamics replaced by this regularized dynamics. In fact the blobs remain localized around their center of vorticity, and hence around $z_{i}(t)$. Moreover we can choose $\varepsilon$ so small that the minimal distance between two blobs is larger than $b / 2$. Finally, by the choice of $\eta=\frac{b}{10}$, we argue that this regularized dynamics coincide with the real one. This concludes the proof.

\section{Generalizations}

First we note that we have studied the problem in the whole $\mathbb{R}^{2}$ only for sake of simplicity, but all the results hold also in a region with boundary. Of course in this case we must consider initial data for which the point vortices never develop collapses or hit the boundary up to the time $T$.

In the present paper we have proved that the "localization" property is a sufficient condition for a rigorous justification of the point vortex model. We can ask whether 
such a derivation is still possible without localization. When all the vortices have intensity of the same sign we can prove the following more general theorem:

Theorem 4.1. Consider an initial datum of the form

$$
\omega_{\varepsilon}(x, 0)=\sum_{i=1}^{N} \omega_{\varepsilon ; i}(x, 0)
$$

where $\omega_{\varepsilon ; 2}(x, 0) \in L_{1}\left(\mathbb{R}^{2}\right) \cap L_{\infty}\left(\mathbb{R}^{2}\right), \omega_{\varepsilon ; i}(x, 0) \geq 0$ and

$$
\int \omega_{\varepsilon ; i}(x, 0) d x=a_{\imath}=\text { vortex intensity. }
$$

Moreover for any bounded continuous function $f$,

$$
\lim _{\varepsilon \rightarrow 0} \int f(x) \omega_{\varepsilon}(x, 0) d x=\sum_{i=1}^{N} a_{\imath} f\left(z_{\imath}\right)
$$

Then

$$
\lim _{\varepsilon \rightarrow 0} \int f(x) \omega_{\varepsilon}(x, t) d x=\sum_{i=1}^{N} a_{i} f\left(z_{i}(t)\right)
$$

where $\omega_{\varepsilon}(x, t)$ is the Euler evolution of the initial datum $\omega_{\varepsilon}(x, 0)$ and $z_{\imath}(t)$ is the solution of the vortex equation (2.20) with initial datum $z_{i}$.

We do not give here the explicit proof, which is essentially the same as in [5] (see also the technique of [8]).

When $a_{i}$ have different sign we do not have, at present, a result like the previous one. However we can generalize the result of the previously section supposing that

$$
\int \omega_{\varepsilon ; i}(x, 0) d x \underset{\varepsilon \rightarrow 0}{\longrightarrow} a_{\imath}
$$

and $\omega_{\varepsilon ; i}(x, 0)$ is bounded outside $\Sigma\left(z_{i} \mid \varepsilon\right)$. Then we consider this last vorticity as the source of an external field that turns out to be uniformly "quasi-Lipschitz." We recall that a bounded vorticity $\omega(y)$ produces a velocity field such that

$$
\int d y\left|K(x-y)-K\left(x^{\prime}-y\right)\right| \omega(y) \leq \operatorname{const}\left(\|\omega\|_{\infty}+\|\omega\|_{1}\right) \phi\left(\left|x-x^{\prime}\right|\right)
$$

where

$$
\varphi(r)=\left\{\begin{array}{ll}
r(1-\ln r) & \text { if } r<1 \\
1 & \text { otherwise }
\end{array} .\right.
$$

Hence, following the same lines of Sect. 3, we can prove: Theorem 4.2. Let $\omega_{\varepsilon}(x, 0)=\sum_{i=1}^{N} \omega_{\varepsilon ; i}(x, 0)$, where $\omega_{\varepsilon ; i}(x, 0)$ have a definite sign and
is such that

$$
\begin{gathered}
\int \omega_{\varepsilon ; i}(x, 0) d x \underset{\varepsilon \rightarrow 0}{\longrightarrow} a_{i}, \\
\left|\omega_{\varepsilon ; i}(x, 0)\right| \leq \mathrm{const} \varepsilon^{-\eta}, \quad \eta<\frac{8}{3}, \\
\left|\omega_{\varepsilon ; i}(x, 0)\right| \leq \mathrm{const} \quad \text { if } x \in \Sigma^{c}\left(z_{\imath} \mid \varepsilon\right), \\
\lim _{\varepsilon \rightarrow 0} \int d x \omega_{\varepsilon}(x, 0) f(x)=\sum_{i=1}^{N} a_{\imath} f\left(z_{\imath}\right),
\end{gathered}
$$


for any continuous bounded function $f$. Then

$$
\lim _{\varepsilon \rightarrow 0} \int d x f(x) \omega_{\varepsilon}(x, t)=\sum_{i=1}^{N} a_{i} f\left(z_{i}(t)\right),
$$

where $\omega_{\varepsilon}(x, t)$ is the Euler evolution of the initial datum $\omega_{\varepsilon}(x, 0)$ and $z_{i}(t)$ is the solution of the vortex equation (2.20) with initial datum $z_{\imath}$.

\section{Vortex-Wave System}

The technique we use for proving Theorem 2.1 gives us also a rigorous justification of the so-called vortex-wave system. This is a model in which point vortices (vortex) and a smooth flow (wave) coexists. More precisely, we consider the initial value problem in $\mathbb{R}^{2}$ :

$$
\begin{gathered}
\frac{d}{d t} \phi_{t}(x)=u\left(\phi_{t}(x)\right)+\sum_{i=1}^{N} \alpha_{i} K\left(\phi_{t}(x)-z_{i}\right) \quad x \neq z_{\imath}(0), \\
\frac{d}{d t} z_{i}(t)=u\left(z_{i}(t), t\right)+\sum_{j=1 ; j \neq i}^{N} \alpha_{j} K\left(z_{i}(t)-z_{j}(t)\right), \\
u(\cdot, t)=(K * \omega)(\cdot, t), \\
\omega\left(\phi_{t}(x), o\right)=\omega_{0}(x) \in L_{1} \cap L_{\infty}, \quad \phi_{0}(x)=x, z_{i}(0)=z_{i} .
\end{gathered}
$$

It describes a system composed by $N$ point $z_{i}$, each of them moving along the velocity field produced by the others and by an incompressible fluid with a bounded vorticity. At the same time this fluid moves along the whole velocity field. When the point vortices are absent this system reduces to the usual Euler equation [notice that here we denote by $\phi_{t}(x)$ the quantity that in Eq. (2.9) we indicated by $\left.x\left(x_{0}, t\right)\right]$. The system (5.1) has been studied in [9], where a global existence theorem has been proved. Moreover with the additional hypothesis that:

$$
\operatorname{supp} \omega_{0}(x) \cap z_{i}=0 \text { for any } i \text { and } z_{i} \neq z_{\jmath} \text { if } i \neq j,
$$

the uniqueness of the solutions and their regularity have also been proved.

We can justify this model by a result analogous of Theorem 2.1:

Theorem 5.1 Consider an initial datum of the form

$$
\omega_{\varepsilon}(x, 0)=\omega_{0}(x)+\sum_{i=1}^{N} \omega_{\varepsilon ; i}(x, 0)
$$

where $\omega_{\varepsilon ; i}(x, 0)$ is a function with a definite sign supported in a region $\Lambda_{\varepsilon ; i}$ such that

$$
\Lambda_{\varepsilon ; i} \equiv \operatorname{supp} \omega_{\varepsilon ; i} \subset \Sigma\left(z_{i} \mid \varepsilon\right) ; \quad \Sigma\left(z_{i} \mid \varepsilon\right) \cap \Sigma\left(z_{j} \mid \varepsilon\right)=\emptyset \quad \text { if } i \neq j
$$

for $\varepsilon$ small enough and where $\Sigma(z \mid r)$ is the circle of center $z$ and radius $r$. Moreover

$$
\text { distance }\left(\cup z_{i} ; \omega_{0}(x)\right)>r_{0} \text { fixed }
$$

and

$$
\int d x \omega_{\varepsilon ; i}(x, 0)=a_{i} \in \mathbb{R} \quad(\text { vortex intensity) }
$$


and

$$
\left|\omega_{\varepsilon ; 2}(x, 0)\right| \leq \text { const } \varepsilon^{-\eta} \quad \eta<\frac{8}{3} .
$$

Then for all $d>0, d \ll r_{0}$ there exists $\varepsilon_{0}(d, T)$ such that, if $\varepsilon<\varepsilon_{0}(d, T)$, then

$$
\operatorname{supp} \omega_{\varepsilon ; i}(x, t) \subset \Sigma\left(z_{i}(t) \mid d\right) \text { for any } t \in[0, T]
$$

and

$$
d \rightarrow 0 \quad \text { as } \quad \varepsilon \rightarrow 0
$$

Moreover for any continuous bounded function $f(x)$

$$
\lim _{\varepsilon \rightarrow 0} \int d x \omega_{\varepsilon}(x, t) f(x)=\int d x \omega\left(\Phi_{t}(x), t\right) f(x)+\sum_{i=1}^{N} a_{i} f\left(z_{i}(t)\right),
$$

where $\omega_{\varepsilon}(x, t)$ is the time evolution of $\omega_{\varepsilon}(x, 0)$ via the Euler equation and $\omega\left(\Phi_{t}(x), t\right)$, $z_{\imath}(t)$ are the solution of the vortex-wave system (5.1).

The proof is analogous to that of Theorem 2.1.

\section{References}

1. Helmholtz, H.: On the integrals of the hydrodynamical equations which express vortex motion. Phil. Mag. 33, 485 (1867);

Kirchhoff, G.: Vorlesungen über Math. Phys., Leipzig: Teuber 1876

Poincaré, H.: Théories des Tourbillons. Paris: George Carré 1893

Kelvin, J.: Mathematical and physical papers. Cambridge: Cambridge University Press 1910

2. Marchioro, C., Pulvirenti, M.: Vortex methods in two-dimensional fluid mechanics. Lecture Notes in Physics, Vol. 203, Berlin, Heidelberg, New York: Springer 1984

3. Marchioro, C., Pulvirenti, M.: Mathematical Theory of incompressible non-viscous fluids. Berlin, Heidelberg, New York: Springer (to appear)

4. Marchioro, C., Pulvirenti, M.: Euler evolution for singular data and vortex theory. Commun. Math. Phys. 91, 563 (1983)

5. Marchioro, C.: Euler evolution for singular initial data and vortex theory: a global solution. Commun. Math. Phys. 116, 45 (1988)

6. Turkington, B.: On the evolution of a concentrated vortex in an ideal fluid. Arch. Rat. Mech. An. 97, 75 (1987)

7. Marchioro, C., Pagani, E.: Evolution of two concentrated vortices in a two-dimensional bounded domain. Math. Meth. Appl. Sci. 8, 328 (1986)

8. Marchioro, C.: On the vanishing viscosity limit for two-dimensional Navier-Stokes equations with singular initial data. Math. Meth. Appl. Sci. 12, 463 (1990)

9. Marchioro, C., Pulvirenti, M.: On vortex-wave system. Mechanics, analysis, and geometry: 200 years after Lagrange. M. Francaviglia (ed.), Amsterdam: Elsevier Science 1991 
